\title{
Dietary fibre intake and common mental disorder: prospective findings from the Whitehall II study
}

\author{
A. Knüppel, M.J. Shipley and E.J. Brunner \\ Department of Epidemiology and Public Health, University College London, London, WC1E 6BT, UK
}

Several pathways potentially link the gut microbiome with common mental disorder ${ }^{(1)}$. Dietary fibre intake is essential in maintaining and shaping the composition of gut microbiome ${ }^{(2)}$. Existing evidence from prospective cohort studies of dietary fibre intake and mood disorders is mixed ${ }^{(3,4)}$. We studied associations between dietary fibre intake and common mental disorder (CMD) in the Whitehall II cohort study. We hypothesised an inverse association of fibre intake and incident common mental disorder 5 years later.

Analysis included 15,487 person-observations collected over 22 years of repeated data-collection. Dietary fibre intake was assessed using food frequency questionnaires, and energy adjusted ${ }^{(5)}$. CMD was measured with the 30-item General Health Questionnaire. Random-effects models were used to model the association between dietary fibre intake and CMD over 5-year cycles. CMD cases $(\geqslant 5$ symptoms) were excluded at the start of each cycle. Analyses were adjusted for potential confounders (shown in Table 1), BMI, central obesity, cardiovascular disease (CVD), diabetes and cancer.

Table 1. Adjusted associations between dietary fibre intake and common mental disorders 5 years later.

\begin{tabular}{|c|c|c|c|c|}
\hline & \multicolumn{4}{|c|}{ Incident common mental disorder at 5 years follow-up } \\
\hline & events / person observations & $\begin{array}{c}\text { Model } 0^{1} \\
\text { OR }(95 \% \mathrm{CI})\end{array}$ & $\begin{array}{c}\text { Model 12 } \\
\text { OR }(95 \% \text { CI })\end{array}$ & $\begin{array}{c}\text { Model } 2^{3} \\
\text { OR }(95 \% \text { CI })\end{array}$ \\
\hline \multicolumn{5}{|l|}{ Dietary fibre intake ${ }^{4}$} \\
\hline Lowest Tertile & $721 / 4941$ & Reference & Reference & Reference \\
\hline Middle Tertile & $658 / 5477$ & $0 \cdot 82 * *(0 \cdot 72,0 \cdot 93)$ & $0 \cdot 82 * *(0 \cdot 71,0 \cdot 93)$ & $0.83 *(0 \cdot 72,0.96)$ \\
\hline Highest Tertile & $577 / 5069$ & $0.76^{* * *}(0.66,0.87)$ & $0.75 * * *(0 \cdot 65,0.86)$ & $0.74 * * *(0.62,0.88)$ \\
\hline$P$ for trend & & $<0.001$ & $<0.001$ & 0.001 \\
\hline $\begin{array}{l}\text { Continuous } \\
(10 \mathrm{~g} / \text { day increment })\end{array}$ & $1956 / 15487$ & $0.87 * * *(0.80,0.94)$ & $0 \cdot 86^{* * *}(0 \cdot 79,0.93)$ & $0.86^{* *}(0.78,0.95)$ \\
\hline
\end{tabular}

${ }^{1}$ Model 0: age, sex, their interaction and ethnicity.

${ }^{2}$ Model 1: Model 0 additionally adjusted for marital status, last grade level in civil service, smoking, alcohol intake, physical activity, sleep duration.

${ }^{3}$ Model 2: Model 1 additionally adjusted fish intake, modified dash score, coffee and tea intake, sugar intake from sweet food/beverages, sugar intake from sweet food/ beverages * sex and total calories.

${ }^{4}$ Dietary fibre intake was energy adjusted.

There was a significant inverse association between dietary fibre intake and CMD (Table $1 ; * \mathrm{P}<0 \cdot 05, * * \mathrm{P}<0 \cdot 01, * * * \mathrm{P}<0 \cdot 001$ ). This association was slightly attenuated by adjustment for socio-economic factors, health behaviours, other dietary intakes such as sugar intake from sweet food/beverages. Further adjustment for BMI, central adiposity, cardio-vascular disease, diabetes and cancer did not change the results (not shown).

The study findings indicate a protective role of a diet high in fibre in long-term psychological health. In conclusion two of three prospective studies support the hypothesis ${ }^{(3,4)}$.

1. Rieder R, Wisniewski PJ, Alderman BL \& Campbell SC (2017) Brain Behav Immun 66, 9-17.

2. Simpson HL \& Campbell BJ (2015) Aliment Pharmacol Ther 42, 158-179.

3. Gangwisch JE, Hale L, Garcia L, Malaspina D, Opler MG, Payne ME, Rossom RC \& Lane D (2015) Am J Clin Nutr 102, $454-463$.

4. Sanchez-Villegas A, Zazpe I, Santiago S, Perez-Cornago A, Martinez-Gonzalez MA \& Lahortiga-Ramos F (2017) Br J Nutr 119, $211-221$.

5. Brunner E, Stallone D, Juneja M, Bingham S \& Marmot M (2001) Br J Nutr 86, 405. 